
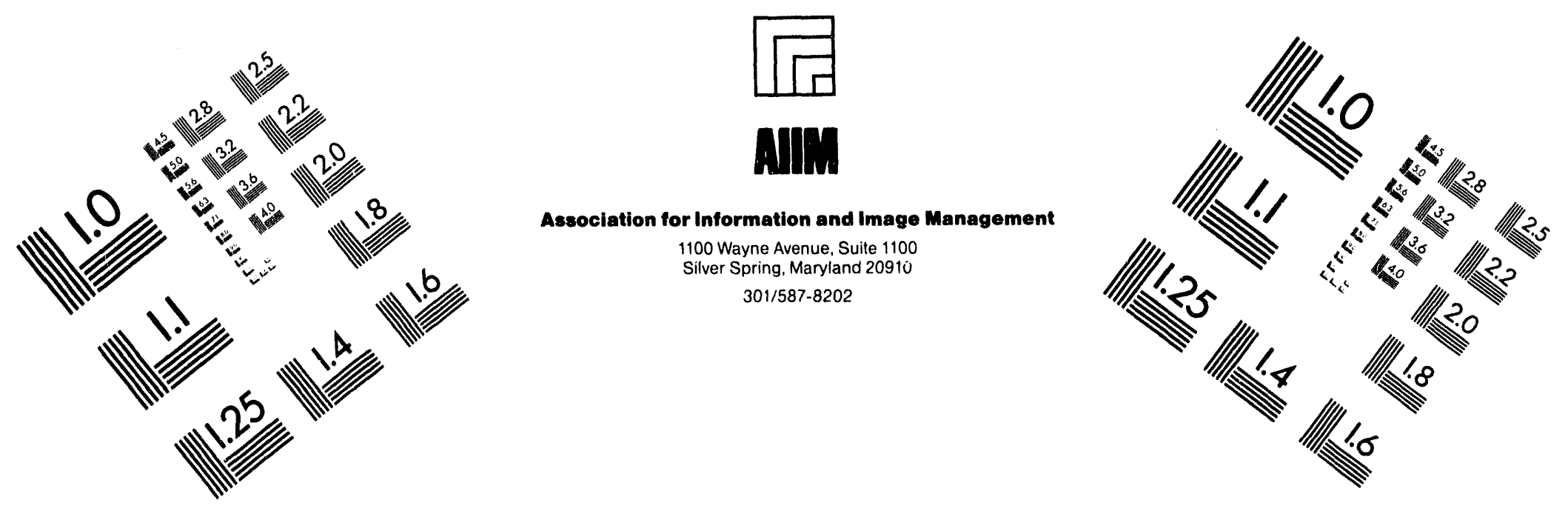

\title{
Centimeter
}

$\begin{array}{llllllllllllllll}1 & 2 & 3 & 4 & 5 & 6 & 7 & 8 & 9 & 10 & 11 & 12 & 13 & 14 & 15 & \mathrm{~mm}\end{array}$ Lшц

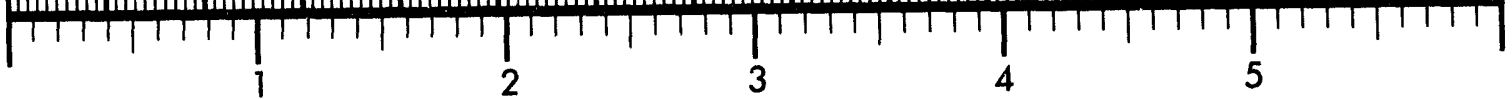
Inches
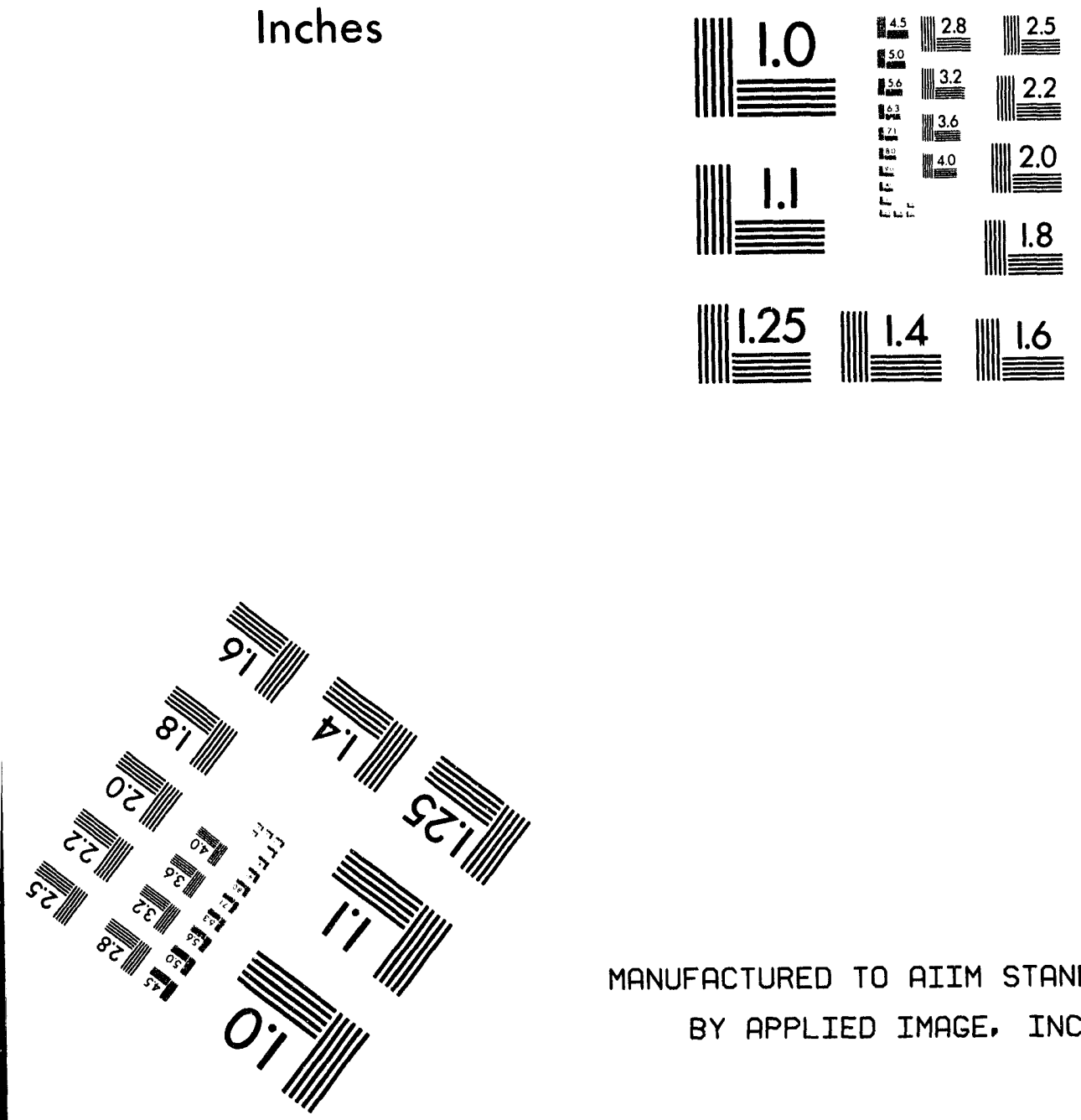

MANUFACTURED TO AIIM STANDARDS BY APPLIED IMAGE, INC.

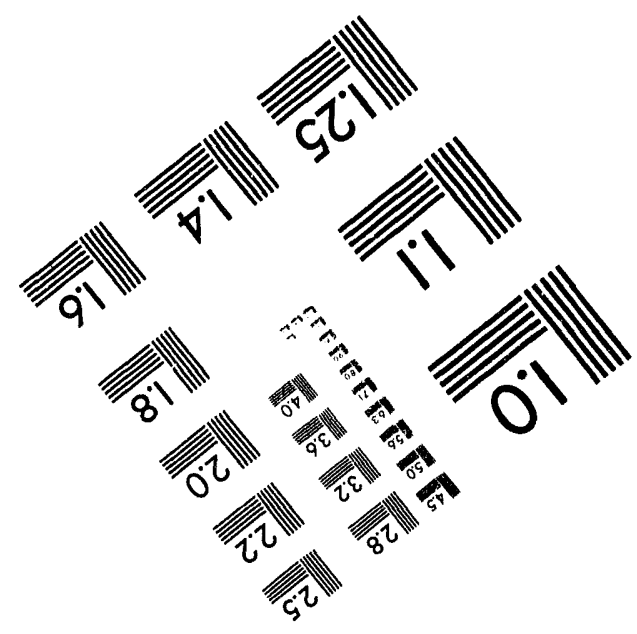



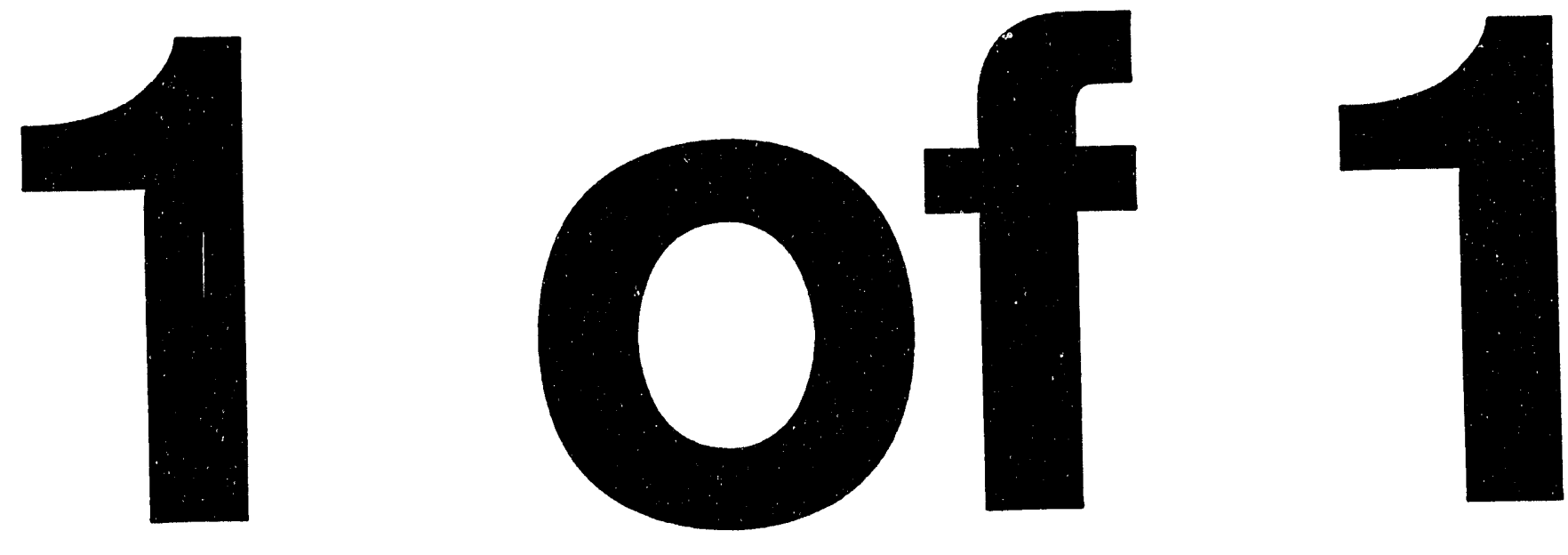
BNL-60710

INFORMAL REPORT

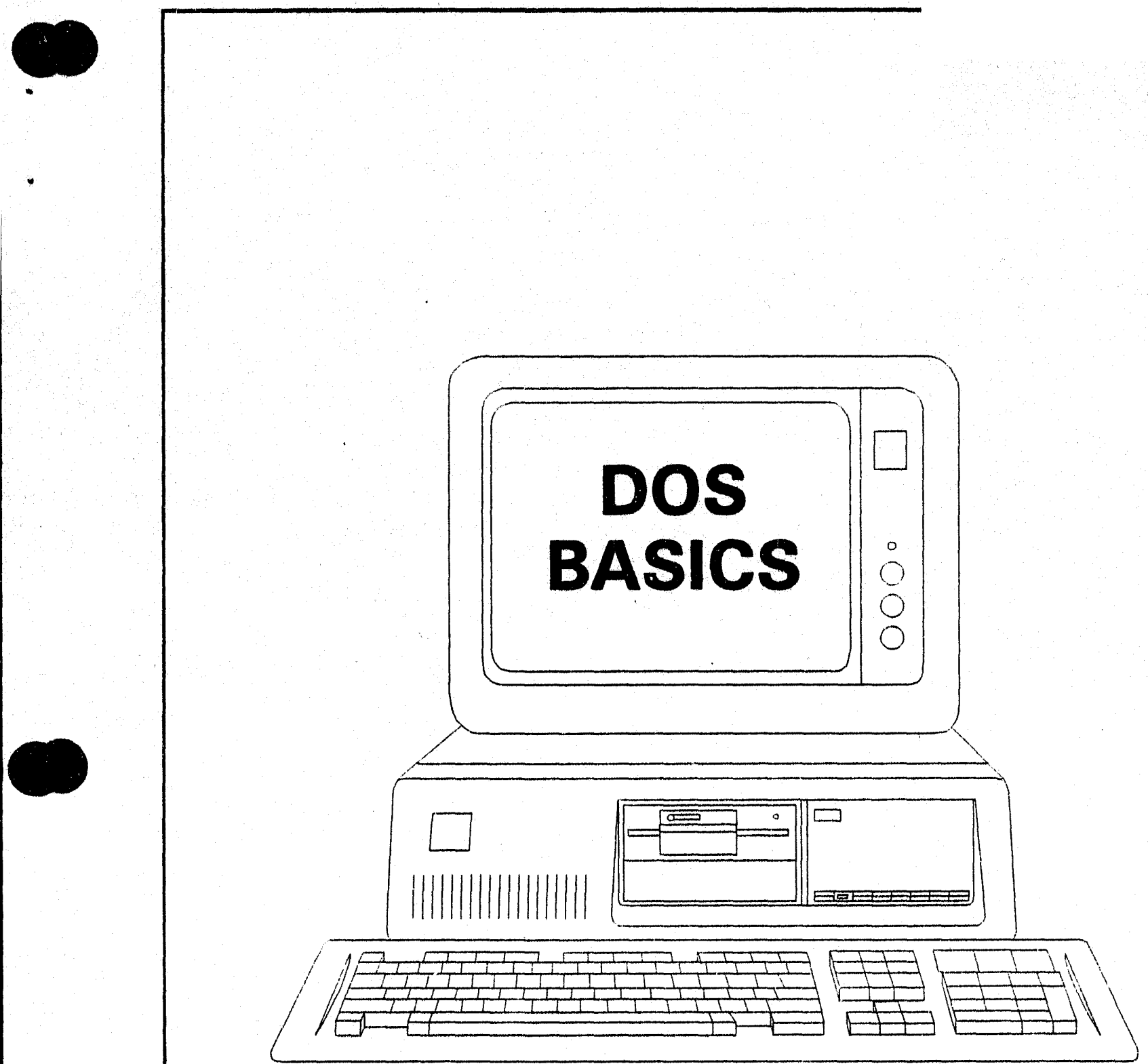

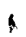

BROOKHAVEN MATIONAL LABORATOAY

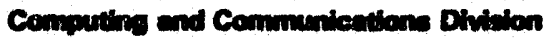
Pec O'Conner, x-7341 


\section{WHAT IS DOS?}

DOS is an acronym for Disk Operating System. It is actually a set of programs that allows you to control your personal computer. DOS offers the capabilities to create and manage files; organize and maintain information placed on disks; use application programs such as WordPerfect, Lotus 123, Excel, Windows, etc. In addition, DOS provides the basic utilities needed to copy files from one area to another, delete files and list files. The latest version of DOS also offers more advanced features that include hard disk compression and memory management.

\section{TERMS YOU SHOULD KNOW:}

BOOT: BOOTing a personal computer, in the most simple terms, means turning it on. A cold boot is initialized when the PC's power switch is turned to the $O N$ position. As the PC boots up, all the configured components within the system are checked as DOS is loaded into memory. A warm boot will reset a PC that is already turned on. Simultaneously pressing the $\langle$ Ctrl $\rangle\langle$ Alt $\rangle$ and $\langle$ Del $\rangle$ keys will initialize the warm boot.

CPU: The Central Processing Unit is the "brains" of the computer. It contains the devices configured in your system such as its hard drive, floppy drive(s) and memory.

HARD DRIVE: Sometimes referred to as a "fixed disk", the hard drive is permanently installed in your system's CPU and is used to store personal computer applications and data. The hard drive is most often designated as the C: drive.

FLOPPY DRIVE: Compartment(s) in which diskettes are loaded. Commonly referred to as the $A$ : and/or $B$ : drive(s).

DISKETTE: "Portahle" disk used for permanent storage of information. Diskettes are commonly $3-1 / 2 "$ and $5-1 / 4 "$ in size.

MEMORY: The element of the CPU that is used as temporary storage for programs and data. The most important component of your PC's memory is RAM. Random Access Memory acts a temporary storage area for your PC applications and data. It is present in your system as long as the PC is turned on. If the information you are working on has not been saved to a permanent storage area (hard disk, floppy disk, diskette) the information will be lost when the PC is turned off!

HARDWARE: The equipment associated with your personal computer: CPU, monitor, keyboard, etc.

\section{DISCLAIMER}

This report was prepared as an account of work sponsored by an agency of the United States Government. Neither the United States Government nor any agency thereof, nor any of their employees, makes any warranty, express or implied, or assumes any legal liability or responsibility for the accuracy, completeness, or usefulness of any information, apparatus, product, or process disclosed, or represents that its use would not infringe privately owned rights. Reference herein to any specific commercial product, process, or service by trade name, tradema manufacturer, or otherwise does not necessarily constitute or imply its endorsement, recommendation, or favoring by the United States Government or any agency thereof. The views and opinions of authors expressed herein do not necessarily state or reflect those of the United States Government or any agency thereof. 
SOFTWARE: The programs and files used and created on your personal computer. Types of software include personal computer applications (WordPerfect, Lotus 123, Windows, etc.) and data files created using PC applications.

PROMPT: A DOS prompt acts as an indicator, displaying the current disk drive and directory designation. Depending upon your personal computer's configuration, this prompt may be displayed specifying the A:, B: or C: drive. The drive specification is usually followed by a greater than sign ( $>$ ) at which a DOS command can be entered.

FILE: A group of information or instructions similar to a memo or file folder.

FILENAME: Filenames can be up to 8 characters long and may contain an optional 3 character extension. This extension must be separated from the filename with a period (eg., MYMEMO.DOC) Filenames and extensions can contain letters and numbers as well as special characters such as \# \& () and . Filenames cannot contain any blank spaces. For example: MY MEMO.TXT would not be acceptable while, MYMEMO.TXT or MY_MEMO.TXT would be just fine!

DIRECTORY: Sections within disks to store similar types of files. Directories are created to better organize and manage the files you install and create on your PC. Subdirectories can be created within directories for even more efficient file maintenance. NOTE: Refer to the directory commands (MD, CD and RD) later in these notes.

PATH: A command's path will specify a DRIVE:IDIRECTORYIFILENAME.EXT that instructs DOS exactly where to find a certain command or file.

DEFAULT: Your personal computer will automatically perform tasks in a certain manner (by default) unless you specifically instruct it to do otherwise within the syntax of your command(s).

BACKUP: Possibly the single most important "personal computer" word. Since the personal computer is yours to manage and control, you must accept the responsibility of maintaining the integrity and security of your files. It is IMPERATIVE that duplications of your files be made on a regular basis in order to insure this integrity and minimize damage to your data. Using the DOS COPY, DISKCOPY and BACKUP commands will help you with this important task. It also may be helpful in preventing those ever-increasing work day frustrations that may lead to ...

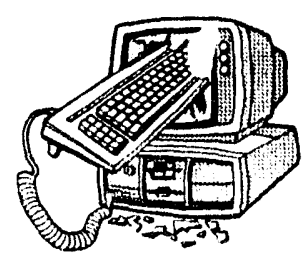

DOS BASICS Pag 2 


\section{THE PERSONAL COMPUTER KEYBOARD}

\begin{tabular}{|c|c|}
\hline KEY & FUNCTION \\
\hline$\leftrightarrow$ Enter & Accept and execute a command \\
\hline $\mathbf{F 1}$ & Repeats last DOS command character by character \\
\hline $\mathbf{F 2}$ & Repeats last DOS command to a specific character \\
\hline F3 & Repeats last DOS command in its entirety \\
\hline F6 & End of file marker (used in EDLIN) \\
\hline Print Screen & "Dump" contents of current screen to printer \\
\hline Scroll Lock & Scrolls cursor screen by screen \\
\hline Pause & Temporarily pauses scrolling \\
\hline Esc & Cancel current command \\
\hline Tab & Advance to next Tab setting \\
\hline Caps Lock & Displays upper case (character keys only) \\
\hline Shift & $\begin{array}{l}\text { In conjunction with other key(s), displays upper case } \\
\text { or symbol noted on top of specified key. }\end{array}$ \\
\hline Ctrl + Break & Aborts command in process \\
\hline Ctrl + Alt + Delete & Warm boot PC \\
\hline Insert & Inserts character(s) to left of cursor position \\
\hline Delete & Deletes character at cursor \\
\hline $\begin{array}{l}\text { Page Up, Page Down, } \\
\text { Home, End }\end{array}$ & "Quick" positions cursor \\
\hline$\uparrow, \downarrow$ & Moves cursor one line up or down \\
\hline$\rightarrow, \leftarrow$ & Moves cursor one character to left or right \\
\hline Backspace & Moves cursor to left, deleting characters \\
\hline Space bar & Moves cursor to right, deleting characters \\
\hline Num Lock & Activates numeric keypad \\
\hline
\end{tabular}




\section{MOVING THROUGH YOUR SYSTEM}

To change the current drive indicator simply type the required drive letter at the current DOS prompt followed by a colon (:) and press the <Enter> key:

Examples:

$\mathrm{C}:>A: \sim$ (displays $A:>$ prompt)

$A:>C: \sim$ (displays $C:>$ prompt)

\section{COMMON DOS COMMANDS}

- FORMAT a diskette

Diskettes cannot be used to store information unless they have been FORMATted first. The FORMAT command must be used with EXTREME CAUTION!! It is, therefore, extremely important to understand just what the FORMAT command does...

- Prepares a diskette (or hard disk) for use. It divides the designated disk into tracks and sectors required by DOS to properly organize files.

- Creates a FAT - File Allocation Table. When a file is retrieved or saved to a disk, the FAT table is read to see exactly where the file resides on the disk.

- eRASes all data/INFormation that may haVe PREVIOUSLY been STORED ON THE DISK! Therefore, think twice before formatting any disk especially the C: drive!

Syntax: FORMAT [DRIVE:]

Example:

C: > FORMAT A: $\triangleleft$ (This entry would format the diskette in the A: drive.) 
- DIR to list files

View a listing a files in the current or designated directory. The DIR command will display each file's name, size and the date it was last modified.

\section{Syntax: DIR [DRIVE:JIPATHIFILENAME.EXT}

$\checkmark$ View all the files in the current directory, example:

$\mathrm{C}:>$ DIR $\leftarrow$

$\checkmark$ View files on another drive, example:

C: > DIR A:

$\checkmark$ List one specific file, example:

C: > DIR FILENAME.EXT

List files with common attributes using DOS' wildcard (*) designator, examples:

$C:>D I R^{*} . T X T \leftrightarrow$ (will display all files on the C: drive with a TXT extension)

C: $>$ DIR A:M*. * $\triangleleft$ (will display all files on the A: drive that begin with the letter "M")

List files and pause at each screen of information, example:

C: $>$ DIR A: /P

$\checkmark$ View files in "widescreen" mode, example:

C: > DIR A: $/ W$

- RENAME a file

Use the rename command to change the name of a file.

Syntax: RENAME [DRIVE:]IPATH|OLDNAME.EXT NEWNAME.EXT

Examples:

C: > RENAME MYTEXT.DOC YOURTEXT.DOC

C: > RENAME A:IOLDMEMO 123 OLDMEMO.456 
- ERASE a file

The erase command will remove the indicated file(s) from the disk or directory specified. Use this command with caution! Erased files cannot be recovered.

\section{Syntax: ERASE [DRIVE:]IPATHIFILENAME.EXT}

Examples:

C: > ERASE YOURTEXT.DOC

C: > ERASE A:IOLDMEMO.456

C: $>$ ERASE A:|MEMOSI*.TXT $\leftarrow$ (This entry would remove all files with a TXT

extension from a directory called MEMOS on the A: drive.)

- COPY a file

The COPY command can be used to copy one or more files from on storage area to another. The COPY command is an invaluable command when used as a tool to backup your files.

\section{Syntax: COPY [DRIVE:]IPATHIFILENAME.EXT [DRIVE:]IPATHIFILENAME.EXT}

Where the file is being copied from (source)?...... Where the file is being copied to (target)?...

$\checkmark$ Copy file(s) from a diskette in floppy drive to a hard disk, example:

C: > COPY A:MYMEMO.DOC C: $\nleftarrow$ (Note: the filename does not have to be specified within the command after the target drive is noted if you plan to retain its original name. An optional new filename, however, can be added. Example:

\section{C: > COPY A:MYMEMO.DOC C:NEWMEMO.DOC $\leftarrow$}

$\checkmark$ Copy file(s) from a hard disk to a diskette in floppy drive, example:

C: $>$ COPY C: ${ }^{*}$.BAT A: $\nleftarrow$ (This entry would copy all files with a BAT extension from the $C$ : drive to the $A$ : drive.)

$\checkmark$ Copy file(s) from one diskette to another, example:

C: > COPY A:MYMEMO.DOC B: $\leftrightarrow$ (HELPFUL HINT: Make sure a formatted diskette is inserted in the target drive before entering this command.)

Copy file(s) from one directory to another within the same disk, example: C: > COPY C:IMEMOSIMYMEMO.DOC C:IOLDSTUFF \& (This entry would copy the file, MYMEMO.DOC from the directory called MEMOS to the directory called, OLDSTUFF. See more about making, changing and removing directories later in these notes.) 
- DISKCOPY the entire contents of one diskette to another diskette.

IMPORTANT: both the source and target diskettes must be the same size.

You cannot, for example, use the DISKCOPY command to copy the entire C: drive to a diskette in the A: drive. In addition, both floppy drives must be configured to accept diskettes of the same density if both are being used in the DISKCOPY command. CAUTION: THIS CUMMAND WILL FORMAT THE TARGET DISKETTE BEFORE IT COPIES THE NEW INFORMATION FROMI THE SOURCE DISKETTE TO IT!

\section{Syntax: DISKCOPY [sourceDRIVE:] [targetDRIVE:]}

Directions:

1. Type DISKCOPY A: A:

2. When prompted, insert the "source" diskette in drive A: and press any key to begin the diskcopy process. Keep in mind that the source diskette contains the information you would like to copy.

3. When prompted, remove the source diskette and insert the "target" diskette in the same drive.

4. When the copy is completed, press Yes to copy the original information to another diskette or No to end the procedure.

- Make a directory (MD)

Use this command to make directories to better organize the application programs you install on your personal computer and the data files you create using these programs. An unlimited number of subdirectories can be nested within directories. IMPORTANT: Make sure your prompt is displaying the drive that you would like to make the directory on.

Syntax: MDINEWDIR

Examples:

C: $>$ MDIMEMOS $\triangleleft$ (This entry would make a directory on the C: drive called, MEMOS.)

C: $>$ MDIMEMOSIMYBOSS $\leftrightarrow$ (This entry would make a second level subdirectory off of the MEMOS directory called, MYBOSS.)

$C:>A:$

A: $>$ MDIPERSONAL $\leftarrow$ (create directory on the diskette in the A: drive called, PERSONAL.) 
- Change directory $(C D)$

The $C D$ command is used to change from one directory to another within a drive.

\section{Syntax: CDIOTHERDIR}

Example:

C: $>$ CDIMEMOS $\leftrightarrow$ (Your prompt should now display, C:IMEMOS $>$ )

$C: \backslash M E M O S>C D M Y B O S S \sim$ (Notice that the backslash $\backslash$ is not used when changing to a subdirectory while the first level directory is noted at the prompt. The new prompt should now display, C:IMEMOSIMYBOSS.)

C:IMEMOSIMYBOSS: $>C D I \hookleftarrow$ (Use the CDI alone to return your prompt to the "root" directory of the current drive. This entry would result in your prompt being returned to $\mathrm{C}:>$

- Remove directory $(R D)$

Use the RD command to remove directories you no longer need. IMPORTANT: a directory cannot be removed if there are still files within it. Therefore, before proceeding with the RD commiand, save the files if you think you'll need them at a later date by copying them to another location or, if you know for sure they are not needed, erase them from the directory you would like to remove. In addition, you prompt cannot indicate that you are currently in the directory you would like to remove. it is often most efficient to use the CDI command to move to the root of the current directory before issuing the RD command.

\section{Syntax: RDIDIRECTORY}

\section{Examples:}

C: $>$ RDIMEMOSIMYBOSS (this would remove the MYBOSS subdirectory. The MEMOS directory would have to be removed in an additional step...)

C: $>$ RDIMEMOS (the MEMOS directory would be removed.) 
- TREE

The TREE command is used to display all of the directories and, optionally, the files within the directories on a designated drive.

\section{Syntax: TREE [DRIVE:]}

Examples:

$C:>$ TREE $\leftarrow$ (Will display the directory structure on the C: drive.)

C: $>$ TREE A: $\longleftarrow$ (Will display the directory structure on the A: drive.)

$C:>$ TREE $/ F \leftrightarrow$ (Will display the directory structure on the $C:$ drive and all files within each directory.)

$\mathrm{C}:>$ TREE /F |MORE $\leftrightarrow$ (Use the |MORE parameter to ensure that the screen will pause as it is filled while listing files.)

- CHKDSK

This command is used to check a designated disk. The FAT table (see the FORMAT command) and directories are checked and analyzed and a status report noting disk size and memory capacity is displayed on your screen. Misallocation and "trouble spots" are noted in the report and, optionally, can be "fixed."

\section{Syntax: CHKDSK [DRIVE:]}

$\mathrm{C}:>C H K D S K \leftarrow$ (The $\mathrm{C}:$ drive will be checked)

C: > CHKDSK A: $\nleftarrow$ (The A: drive will be checked)

C: > CHKDSK A: /F $\leftrightarrow$ (The A: drive will be checked. If found, lost clusters will be converted (fixed) to files with CHK extensions that can be erased. 
- BACKUP the hard drive

Possibly DOS' most importani command, BACKUP will assist you in duplicating those files that reside on your hard drive. Files that are backed up can, of course, be retrieved when and if a hard disk failure ever occurs. IMPORTANT: MAKE SURE THAT YOU HAVE A SUBSTANTIAL NUMBER OF FORMATTED DISKETTES PREPARED AND ON HAND BEFORE INITIATING THIS COMMAND. When initialized, the backup command will offer on-screen prompts directing you when to insert another diskette in the target drive as previous diskettes become filled. Each resulting backup diskette will contain a "saveset" backup file with a corresponding number, depending on the order it was inserted during the backup procedure. For example, your first diskette will contain a "saveset" file called BACKUP.001 which, in effect, contains as many files as the diskette's size would allow during the procedure.

\section{Syntax: BACKUP [sourceDRIVE:] [targetDRIVE:]}

\section{Examples:}

C: $>$ BACKUP C:I A: $\nleftarrow$ (This entry will backup only those files that reside on the root directory of the $C$ : drive to the $A$ : drive.)

C: $>$ BACKUP $C: \mid$ A: $/ S \leftarrow$ (This entry will backup all files on the root as well as those within any directory on the C: drive. The original Structure of the directories and their associated files will be maintained when they are copied to the target drive $A:$ )

C: > BACKUP C:IMEMOS A: $\sim$ (Backup all files in the IMEMOS subdirectory on the C: drive to the target drive $A:)$

C: > BACKUP C:IMEMOS A: /M /A $\leftarrow$ (Backup only those files in the IMEMOS subdirectory on the $\mathrm{C}$ : drive that have been Modified since the last backup procedure and Append them to the end of the backup disks.)

C: > BACKUP C: I A: $/ S / D: 4 / 17 / 94 \leftrightarrow$ (This entry will backup all files on the root of the C: drive for a specific $D$ ate (in this instance, 4/17/94) while maintaining the original directory Structure.) 
- RESTORE backed up files to the hard drive

Files that are backed up using the DOS BACKUP command can only be transferred to your system's hard drive using the RESTORE command. The RESTORE command analyzes the contents of the "saveset" files backed up on a diskette and, depending on the specific parameters you designate in the syntax of the command, restores part or all of the files backed up to the target hard drive. Helpful Hint: Use the DIR command before initializing the RESTORE command to get a listing of the contents of each of the backed up diskettes. Take note of the corresponding backup saveset file number on each diskette. You will be prompted during the RESTORE command to insert the backup diskettes in order.

\section{Syntax: RESTORE [sourceDISKETTE] [targetDRIVE:]}

\section{Examples:}

C: > RESTORE A: C: $/ S \leftrightarrow$ (Restores all files on diskette(s) in A: drive to the C: drive, maintaining the original directory Structure of the files at the time they were backed up.)

C: > RESTORE A: C:IMEMOS $\leftrightarrow$ (This entry would restore only those files in the srecitic subdirectory called MEMOS.)

C: > RESTORE A: C: $\mid$ MEMOS|*.DOC $\backsim$ (This entry would restore only those files with a $D O C$ extension to the MEMOS subdirectory on the $C$ : drive.)

C: > RESTORE A: C: /A:8/15/94 $\nleftarrow$ (This entry would restore only those files created or modified on or After the date, August 15, 1993.)

C: > RESTORE A: C: /B:8/15/94 $\nleftarrow$ (Restore all files created or modified on or Before the date, August 15, 1994.) 


\section{CREATING BATCH FILES}

Automate frequently used DOS commands by storing them in BATCH files! A batch files contains a series of DOS commands that can be executed by simply typing the name of the batch file.

For example, suppose you are a frequent WordPerfect user. The files needed to run WordPerfect may reside in a subdirectory on your C: drive called, WP51. Therefore, to activate the WordPerfect program, you must first use DOS' CD (Change Directory) command to make the WP51 subdirectory current. Once the proper subdirectory is noted at your prompt, the proper "executable" file defined by the software's manufacturer must be invoked. In this example, the executable filed called WP must be entered. Finally, when you are finished with your WordPerfect session and properly exit it, you will be returned to the DOS prompt. The C:IWP51 subdirectory is still displayed at the prompt. You would have to use the CDI command to return your prompt to the root directory. Rather than invoking each of these commands individually, they can be stored in a BATCH file which would interpret each command and invoke them automatically.

- The COPY CON command can used to create a batch file. When entered, your cursor is moved to a blank line beneath the command. Use the $\leftarrow<$ Enter > key after each of the commands you would like to store in the batch file. Finally, press the $\langle F 6\rangle$ function key to end the batch file creation.

\section{Example:}

Using the scenario noted on the previous page, the following keystrokes would be entered to create a batch file called USEWP.BAT that would automatically invoke the WordPerfect program:

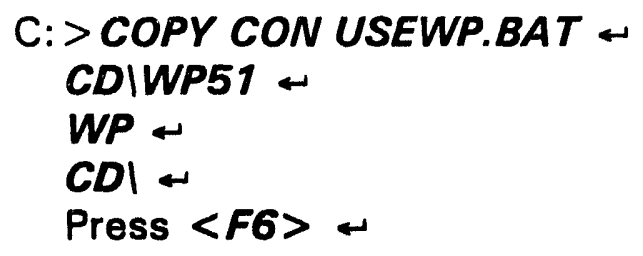

Now, whenever you wnuld like to initialize WordPerfect, you would simply type the following:

$C:>$ USEWP $\leftarrow$ 


\section{CREATING BATCH FILES, cont'd}

- To display the contents of a batch (or other text or ASCII) file that was previously created, use the TYPE command. Examples:
C: > TYPE USEWP.BAT $\leftrightarrow$ (will display the cciltents of the USEWP.BAT file on the screen)
C: $>$ TYPE USEWP.BAT $>$ PRN $\nleftarrow$ (will send the contents of the USEWP.BAT to the printer)

- Each version of DOS comes with a built-in line editor called, EDLIN. (NOTE: Later versions of DOS also come with a more sophisticated line editor.) EDLIN is used to create new or edit existing BATCH files. It can also be utilized to make changes to s:mple text (or ASCII) files.

\section{Directions:}

\section{At the proper directory prompt type EDLIN <FILENAME> and press $\leftarrow$}

2. An asterisk $(*)$ prompt will appear beneath the command. Type the letter "I" and press $\leftarrow$ to display a listing of the commands contained in the designated batch file. Each command listed will be prefaced with the line number it has been entered on. Take note of the line number you would like to edit.

3. To edit a command: simply type the number of the line you would like to edit and press $\sim$. A duplicate of the line will appear on your screen. An asterisk $\left(^{*}\right)$ and a blank area will appear beneath this. Edit the line as required and press $\langle\mathbf{F 6}\rangle$ when you are finished. Your cursor will return to an asterisk (*) prompt at the margin. Edit any other lines as required by entering the appropriate number or type the letter " $\theta$ " to exit EDLIN and save your changes.

To delete a command: type the line number of the command you would like to delete at the asterisk (*) prompt followed by the letter " $d$ " to delete the appropriate line (for example $2 d \leftrightarrow$ will delete line number two from the batch file.) Your cursor will return to an asterisk $(*)$ prompt at the margin. Delete any other lines as required by entering the appropriate number or type the letter "e" to exit EDLIN and save your changes.

To insert a new command: note the line number of the command you would like to insert the new command above. Type this line number at the asterisk $\left({ }^{*}\right)$ prompt followed by the letter " $i$ " to insert a new line. (For example, $3 i \sim$ will allow you to insert a new command above the command noted on line three.) An indented asterisk (*) prompt will appear for you to enter the new command. Press <F6> when completed. The cursor will be returned to the asterisk (") prompt at the margin. Press the letter " $\theta$ " to exit EDLIN and save your changes. 

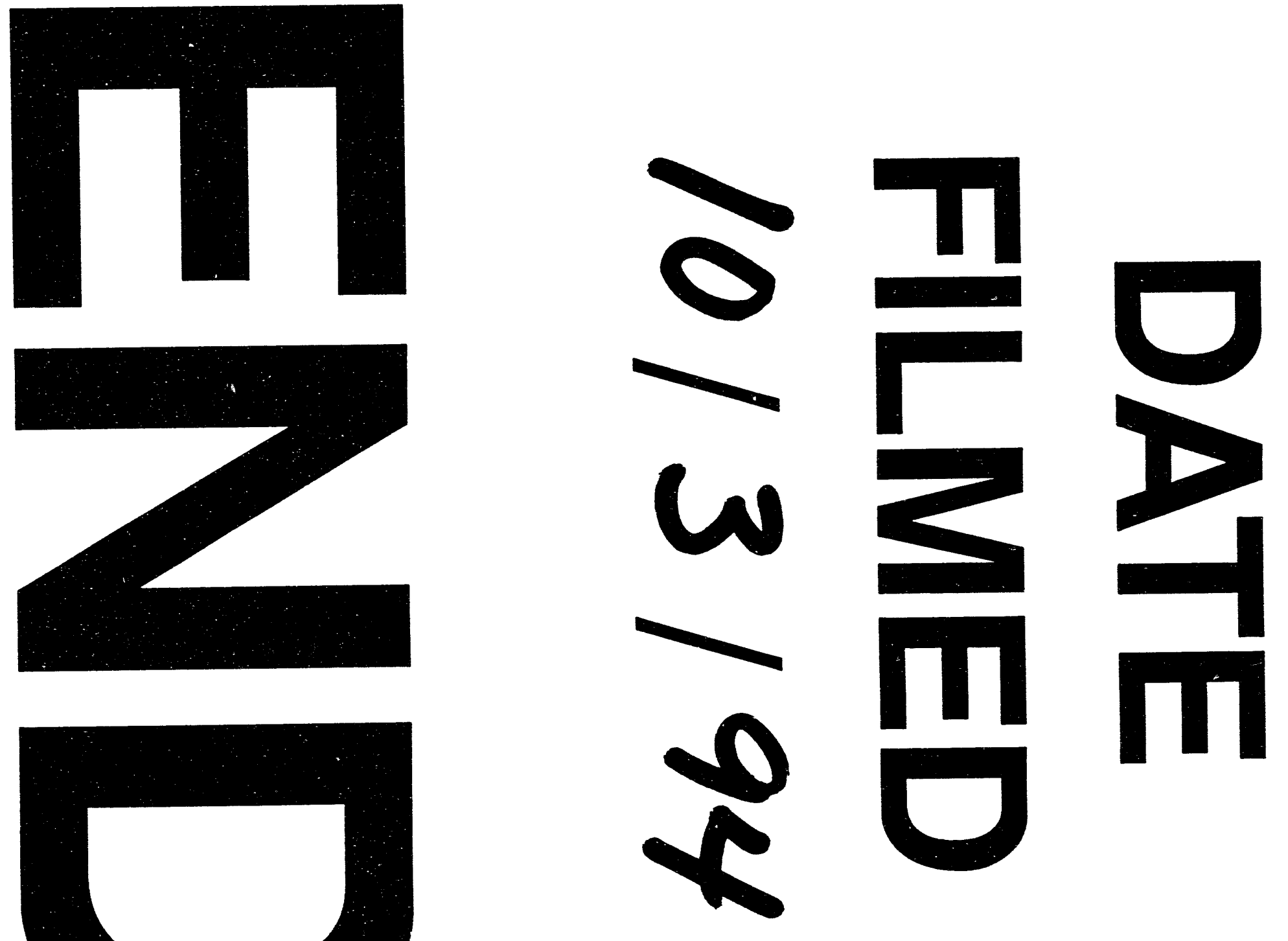
